LAPTH $-1263 / 08$

MPP-2008-88

\title{
Observing trans-Planckian ripples in the primordial power spectrum with future large scale structure probes
}

\author{
Jan Hamann \\ LAPTH (Laboratoire d'Annecy-le-Vieux de Physique Théorique, CNRS UMR5108 \\ \& Université de Savoie), BP 110, F-74941 Annecy-le-Vieux Cedex, France
}

\section{Steen Hannestad, Martin S. Sloth}

Department of Physics and Astronomy, University of Aarhus

Ny Munkegade, DK-8000 Aarhus C, Denmark

\section{Yvonne Y. Y. Wong}

Max-Planck-Institut für Physik (Werner-Heisenberg-Institut)

Föhringer Ring 6, D-80805 München, Germany

E-mail: hamann@lapp.in2p3.fr, sth@phys.au.dk, sloth@phys.au.dk, ywong@mppmu .mpg.de

\begin{abstract}
We revisit the issue of ripples in the primordial power spectra caused by trans-Planckian physics, and the potential for their detection by future cosmological probes. We find that for reasonably large values of the first slow-roll parameter $\epsilon$ ( $\gtrsim 0.001$ ), a positive detection of trans-Planckian ripples can be made even if the amplitude is as low as $10^{-4}$. Data from the Large Synoptic Survey Telescope (LSST) and the proposed future $21 \mathrm{~cm}$ survey with the Fast Fourier Transform Telescope (FFTT) will be particularly useful in this regard. If the scale of inflation is close to its present upper bound, a scale of new physics as high as $\sim 0.2 M_{\mathrm{P}}$ could lead to observable signatures.
\end{abstract}




\section{Introduction}

Observations of the temperature and polarisation anisotropies in the cosmic microwave background $(\mathrm{CMB})$ radiation strongly support the idea that the initial conditions for structure formation were set by an earlier period of cosmic inflation [1-4]. During this period, the rapid expansion of space caused quantum fluctuations of the inflaton field to be blown up to macroscopic scales. The initial state of these quantum fluctuations is typically assumed to be given by the Bunch-Davies vacuum of de Sitter space at a time when $k \gg a H$, i.e., the physical wavenumber $k / a$ is much larger than the Hubble expansion rate. Along with the usual assumptions of single-field, slowroll inflation, this leads to the almost scale-invariant spectrum of Gaussian adiabatic scalar perturbations that constitutes the "primordial" state of perturbations in today's cosmological concordance model [5].

However, in an expanding space-time the notion of a vacuum is not unique. This can easily be understood by observing that the expanding space-time makes the Hamiltonian depend explicitly on time, so there is no time-independent lowest energy state that can serve as a vacuum at all times. We might then worry about how sensitive our predictions are to the choice of initial state. The choice of vacua other than the Bunch-Davies one leads in principle to unacceptable ultraviolet divergences in the observables [6], although this deficiency can be cured by introducing a high-energy cutoff to the theory. In practice, such a cutoff will be given by the Planck scale, or possibly even lower, by a scale of new physics $\Lambda$ (if the cutoff is too close to the Planck scale, an unorthodox vacuum choice may in fact lead to backreaction problems [7-12]). An ultraviolet cutoff in the inflaton fluctuation modes violates energy conservation and has to be associated with a source term for the fluctuations, in order to account for the modes redshifting across the new physics hypersurface [10].

Unless inflation started only just before the wavelengths corresponding to today's observable scales left the horizon, these fluctuations can be mapped to wavenumbers larger than $\Lambda$ during inflation and have emerged from above the new physics hypersurface. It is thus very well conceivable that the fluctuations bear an imprint of the unknown new physics $[13,14]$. In particular, the modes might be created in a non-standard vacuum state at the new physics hypersurface. Danielsson proposed a prescription of how to construct this initial state by minimising the Hamiltonian on this hypersurface [15]. However, it was found in references [16,17] that the state constructed in this fashion is somewhat ambiguous, since it is not invariant under canonical transformations of the Hamiltonian. In fact, it has been argued from a purely effective field theory point of view that the effect on the primordial spectrum should be smaller than that proposed by Danielsson [18]. Eventually, only a better understanding of the thinning of ultraviolet degrees of freedom in quantum gravity [19] can help us solve the puzzle of the correct choice of initial conditions and how the fluctuations emerge during inflation, as in the proposed trans-Planckian damping mechanism [20] (see also [9]). 
Nonetheless, the generic signature of choosing a non-Bunch-Davies vacuum state appears to be a superimposed oscillation of the primordial spectra ("ripples"), whose amplitude is suppressed by a power of $H / \Lambda$. For definiteness we will consider Danielsson's model as a case study in the following.

The question of whether traces of these oscillations can possibly be detected in present or future CMB anisotropy data has been the subject of a number of recent studies [21-30]. While few will disagree that the CMB anisotropies are at present the most powerful probe of the primordial power spectrum, the advent of high-redshift surveys of the large scale structure (LSS) distribution in the not-so-distant future may help to enlarge the detection window. Planned galaxy redshift surveys such as the Large Synoptic Survey Telescope (LSST) [31], or surveys of the distribution of neutral hydrogen using the $21 \mathrm{~cm}$ spin-flip line (e.g., the Murchison Widefield Array (MWA) [32], the Square Kilometre Array [33], or the Fast Fourier Transform Telescope (FFTT) [34]) have the advantage of mapping out the density perturbations in three dimensions over large volumes. They are less affected by sampling variance (or cosmic variance), which is ultimately the limiting factor for CMB data over a large range of scales. In this work, we investigate how a combination of these probes with CMB data can enhance our potential to discover the footprints of new physics beyond the energy scale of inflation.

We shall begin by refreshing the reader's memory of the Danielsson model in section 2. Some general considerations about the phenomenology of oscillating spectra will be presented in section 3 , before we turn to the questions of under which conditions their traces can be detected in CMB data (section 4), or, if no detection is possible, what constraints can be placed on the parameters of the model (section 5). In sections 6 and 7 we discuss how future galaxy redshift surveys and $21 \mathrm{~cm}$ surveys can contribute to improving the chances for a detection of ripples. We conclude in section 8 .

\section{The model}

The spectrum of CMB perturbations predicted from inflation is usually expressed in terms of the spectrum of co-moving curvature perturbations, since this is a conserved quantity on super-Hubble scales in single field slow-roll inflation. In the spatially flat gauge, the curvature perturbation $\mathcal{R}(k)$ can be related to the fluctuation of the inflaton field $\delta \phi(k)$ by the relation $\delta \phi(k)=(\dot{\phi} / H) \mathcal{R}(k)$, where $H$ is the Hubble scale and $\phi$ is the classical expectation value of the inflaton field.

Let us remind the reader of the Danielsson initial condition and at the same time generalise the derivation of the spectra to the case of standard slow-roll inflation. Since there has been some confusion about the correct generalisation to slow-roll in the literature, we repeat some of the details.

In the spatially flat gauge we can write the metric in the form

$$
d s^{2}=a^{2}(\tau)\left(d \tau^{2}-d x^{2}\right),
$$

and after transforming to the canonical variable $\mu=a \delta \phi$, the perturbation of the inflaton 
field satisfies the Mukhanov equation, which takes the form of the equation of motion of a harmonic oscillator with a time-dependent mass [5],

$$
\mu_{k}^{\prime \prime}+\left[k^{2}-\frac{1}{\tau^{2}}\left(\nu^{2}-\frac{1}{4}\right)\right] \mu_{k}=0
$$

where $\nu=3 / 2+3 \epsilon-\eta$, and $\epsilon$ and $\eta$ are the usual slow-roll parameters which we assume to be constant. The conjugate momentum to $\mu_{k}$ is given by [5]

$$
\pi_{k}=\mu_{k}^{\prime}-\frac{z^{\prime}}{z} \mu_{k}
$$

where $z$ is defined as $z \equiv \sqrt{2 \epsilon} a$.

Like for the ordinary harmonic oscillator, it is convenient to quantise the system by promoting the fields to operators and expanding them in terms of raising and lowering operators

$$
\begin{aligned}
& \hat{\mu}_{k}(\tau)=\frac{1}{\sqrt{2 k}}\left[\hat{a}_{k}(\tau)+\hat{a}_{-k}^{\dagger}(\tau)\right], \\
& \hat{\pi}_{k}(\tau)=-i \sqrt{\frac{k}{2}}\left[\hat{a}_{k}(\tau)-\hat{a}_{-k}^{\dagger}(\tau)\right],
\end{aligned}
$$

where the vacuum is defined as the state annihilated by the lowering operator

$$
\hat{a}_{k}(\tau)|0\rangle_{\tau}=0 .
$$

Now, the vacuum at any later time is determined by a Bogoliubov transformation

$$
\begin{aligned}
& \hat{a}_{\mathbf{k}}(\tau)=\alpha_{k}(\tau) \hat{a}_{\mathbf{k}}\left(\tau_{0}\right)+\beta_{k}(\tau) \hat{a}_{-\mathbf{k}}^{\dagger}\left(\tau_{0}\right), \\
& \hat{a}_{\mathbf{k}}^{\dagger}(\tau)=\alpha_{k}^{*}(\tau) \hat{a}_{\mathbf{k}}^{\dagger}\left(\tau_{0}\right)+\beta_{k}^{*}(\tau) \hat{a}_{-\mathbf{k}}\left(\tau_{0}\right),
\end{aligned}
$$

where $\alpha_{k}$ and $\beta_{k}$ are the Bogoliubov coefficients satisfying the normalisation condition

$$
\left|\alpha_{k}\right|^{2}-\left|\beta_{k}\right|^{2}=1
$$

in order for the commutation relations to be preserved in time. The solution to the dynamical equations is most easily found by defining a set of mode functions

$$
\begin{aligned}
& f_{k}(\eta)=\frac{1}{\sqrt{2 k}}\left[\alpha_{k}(\tau)+\beta_{k}^{*}(\tau)\right], \\
& g_{k}(\tau)=-i \sqrt{\frac{k}{2}}\left[\alpha_{k}(\eta)-\beta_{k}^{*}(\tau)\right],
\end{aligned}
$$

which satisfies the classical equation of motion, as can be seen by employing the Heisenberg equation of motion. One can then invert these relations to find

$$
\begin{aligned}
& \mu_{k}(\eta)=f_{k}(\eta) a_{k}\left(\eta_{0}\right)+f_{k}^{*}(\eta) a_{-k}^{\dagger}\left(\eta_{0}\right), \\
& \pi_{k}(\eta)=g_{k}(\eta) a_{k}\left(\eta_{0}\right)+g_{k}^{*}(\eta) a_{-k}^{\dagger}\left(\eta_{0}\right) .
\end{aligned}
$$

By minimising the Hamiltonian written in terms of the variable $\phi$ and its conjugate momenta (note that minimising the Hamiltonian written in terms of the Mukhanov variable gives a different result $[16,17]$ ), one can show that the instantaneous vacuum $|0\rangle_{\tau_{0}}$ that minimises the energy at $\tau=\tau_{0}$ is determined by

$$
g_{k}\left(\tau_{0}\right)=-i k f_{k}\left(\tau_{0}\right) .
$$


This is equivalent to the zeroth order adiabatic vacuum, and the condition is equivalent to requiring $\beta\left(\tau_{0}\right)=0$, which one can use to normalise the general solution to the Mukhanov equation

$$
\begin{aligned}
f_{k} & =\frac{A_{k}}{\sqrt{2 k}} e^{-i \zeta} \sqrt{-x} H_{\nu}^{(1)}(-x)+\frac{B_{k}}{\sqrt{2 k}} e^{i \zeta} \sqrt{-x} H_{\nu}^{(2)}(-x), \\
g_{k} & =-A_{k} e^{-i \zeta} \sqrt{\frac{k}{2}} \sqrt{-x} H_{\nu-1}^{(1)}(-x)-B_{k} e^{i \zeta} \sqrt{\frac{k}{2}} \sqrt{-x} H_{\nu-1}^{(2)}(-x),
\end{aligned}
$$

where $A_{k}$ and $B_{k}$ are constants of integration to be fixed by the physical initial conditions and $\zeta=-\left(\frac{1}{2} \nu+\frac{1}{4}\right) \pi$. Solving the equations for the Bogoliubov coefficients $\alpha_{k}$ and $\beta_{k}$, and expanding in $1 / x$, one obtains

$$
\begin{aligned}
& \alpha_{k}=\frac{1}{2}\left[A_{k} e^{-i k \eta}\left(2-i \frac{\nu^{2}-2 \nu+1 / 2}{k \eta}\right)+B_{k} e^{i k \eta} i \frac{\nu-1 / 2}{k \eta}\right], \\
& \beta_{k}^{*}=\frac{1}{2}\left[B_{k} e^{i k \eta}\left(2+i \frac{\nu^{2}-2 \nu+1 / 2}{k \eta}\right)-A_{k} e^{-i k \eta} i \frac{\nu-1 / 2}{k \eta}\right] .
\end{aligned}
$$

¿From equation (2.7), we also have

$$
\left|A_{k}\right|^{2}-\left|B_{k}\right|^{2}=1
$$

As mentioned above, the Danielsson choice of vacuum requires that we put $\beta_{k}\left(\tau_{0}\right)=0$ at some initial moment $\tau_{0}$. This implies that

$$
B_{k}=i e^{-2 i k \eta_{0}} \frac{\nu-1 / 2}{2 k \eta_{0}+i\left(\nu^{2}-2 \nu+1 / 2\right)} A_{k},
$$

from which we conclude that

$$
\left|A_{k}\right|^{2}=\frac{1}{1-\left|\chi_{k}\right|^{2}}
$$

where

$$
\chi_{k}=\frac{i(\nu-1 / 2)}{2 k \eta_{0}+i\left(\nu^{2}-2 \nu+1 / 2\right)} .
$$

One can then calculate the spectrum of curvature perturbations $P_{\mathcal{R}}=(1 / 2 \epsilon) P_{\phi}$, which becomes

$$
\begin{aligned}
P_{\phi}= & \frac{1}{a^{2}} P_{\mu}=\frac{k^{3}}{2 \pi^{2} a^{2}}\left|f_{k}\right|^{2} \\
\sim & \frac{2^{2 \nu-3}}{4 \pi^{2} \tau^{2} a^{2}}\left[\frac{\Gamma(\nu)}{\Gamma(3 / 2)}\right]^{2}(-k \tau)^{3-2 \nu}\left(\left|A_{k}\right|^{2}+\left|B_{k}\right|^{2}-A_{k}^{*} B_{k}-A_{k} B_{k}^{*}\right) \\
= & 2^{2 \nu-3}(1-\epsilon)^{2 \nu-1}\left[\frac{\Gamma(\nu)}{\Gamma(3 / 2)}\right]^{2}\left(\frac{H}{2 \pi}\right)^{2} \\
& \times\left(1+\left|\alpha_{k}\right|^{2}-\chi_{k} e^{-2 i k \tau_{0}}-\chi_{k}^{*} e^{2 i k \tau_{0}}\right) \frac{1}{1-\left|\chi_{k}\right|^{2}},
\end{aligned}
$$

where we have taken the super-horizon limit $\tau \rightarrow 0$ and evaluated the expression at horizon crossing $k=a H$, using $\tau=-1 /[a H(1-\epsilon)]$ in the prefactor. Inserting $\chi$ gives, up to a phase,

$$
P_{\mathcal{R}}=P_{\mathcal{R}}^{B D}(k)\left[1-\frac{\nu-1 / 2}{x_{0}} \sin \left(2 x_{0}\right)\right],
$$


where $x_{0}=-k \eta_{0}$.

This expression agrees with the straightforward generalisation of the corresponding calculation for power-law inflation in references $[16,17]$, but disagrees slightly with the result of [35], who found that the expression does not depend on $\eta$. This can be traced to an inaccurate approximation. $f$

Following the proposal of Danielsson, we should fix the initial condition of a given mode when that mode crosses a fixed cutoff, i.e.,

$$
\left|x_{0}\right|=\Lambda / H_{0},
$$

where factors of order one can be absorbed in the arbitrary cutoff $\Lambda$. Here $H_{0}$ is the Hubble parameter at $\tau=\tau_{0}$, when the mode crosses the new physics hypersurface defined by the ultraviolet cutoff $\Lambda$. Since $H_{0}$ is not the same for all modes because of the slowly decreasing $H$, this will introduce $k$-dependent oscillations in the spectrum. It can be shown that this leads to $x_{0} \propto k^{\epsilon}$, since $H_{0} \propto k^{-\epsilon}$ [35]. One can then normalise $H$ to $H_{0}$ to obtain

$$
\left|x_{0}\right|=\frac{\Lambda}{H_{0}}\left(\frac{k}{k_{0}}\right)^{\epsilon}
$$

which leads to

$$
P_{\mathcal{R}}(k)=P_{\mathcal{R}}^{B D}(k)\left(1+(\nu-1 / 2) \xi\left(\frac{k}{k_{0}}\right)^{-\epsilon} \sin \left[\frac{2}{\xi}\left(\frac{k}{k_{0}}\right)^{\epsilon}+\phi\right]\right),
$$

where $\xi \equiv H_{0} / \Lambda$. The phase $\phi$ needs to be added as a free parameter since any dependence of the final result on the (arbitrary) choice of pivot scale $k_{0}$ is non-physical and must necessarily be spurious. A similar expression exists for the tensor power spectrum, i.e., $P_{h}(k)=P_{h}^{B D}(k)(\cdots)$.

\section{Preliminary considerations}

In the limit $\epsilon \ll 1$, the argument of the sine function in equation (2.21) can be expanded in powers of $\epsilon$. To the lowest order in $\epsilon$ and $\eta$,

$$
P_{\mathcal{R}, h}(k) \simeq P_{\mathcal{R}, h}^{B D}(k)\left(1+\xi\left(\frac{k}{k_{0}}\right)^{-\epsilon} \sin \left[\frac{2 \epsilon}{\xi} \ln \left(\frac{k}{k_{0}}\right)+\phi^{\prime}\right]\right),
$$

i.e., the oscillations are in $\ln k$, with frequency $\omega \equiv 2 \epsilon / \xi$, or equivalently, oscillation length $L \equiv \pi \xi / \epsilon$, and we have defined $\phi^{\prime} \equiv \phi+2(1+\epsilon) / \xi$.

$\ddagger$ The procedure outlined in the footnote on page 7 of [35] is slightly misleading. Although the zeroth order adiabatic approximation is not exact, its solution is only used as a boundary condition to normalise the exact solution at the initial time giving the zeroth order adiabatic vacuum solution, which is an

exact solution. In the end we want to end up with an exact solution, otherwise the effect would just be an effect of an inaccurate approximation. 


\subsection{Constraints on the oscillation length}

The totality of CMB and LSS observations spans roughly four orders of magnitude in $k$ space between $k_{\min } \sim 10^{-5} h \mathrm{Mpc}^{-1}$ and $k_{\max } \sim 10^{-1} h \mathrm{Mpc}^{-1}$. In natural log space this range is approximately $\ln \left(k_{\max } / k_{\min }\right) \simeq \ln \left(10^{4}\right) \simeq 9.2$. For the individual experiment we take the observable $k$ range to be that in which the measurements are effectively noise-free and limited only by cosmic variance.

For a CMB survey that is cosmic variance-limited up to $\ell=2000$ (the "CVL survey"), we have $k_{\max } \simeq 0.15 \mathrm{~h} \mathrm{Mpc}^{-1}$ within the concordance $\Lambda \mathrm{CDM}$ framework. The Planck satellite also has similar specifications in its $T T$ measurements [36]. The WMAP $T T$ power spectrum is cosmic variance-limited only up to $\ell \simeq 500$ [37], corresponding to $k_{\max } \simeq 5 \times 10^{-2} h \mathrm{Mpc}^{-1}$. Therefore,

$$
R_{\mathrm{CMB}}=\ln \left(k_{\max } / k_{\min }\right) \simeq \begin{cases}6.9, & \text { WMAP, } \\ 8.0, & \text { Planck and CVL, }\end{cases}
$$

where in both cases we have used $k_{\min } \simeq 5 \times 10^{-5} h \mathrm{Mpc}^{-1}$.

If trans-Planckian corrections to the primordial power spectrum are to be observed as an oscillatory signal, we must be able to fit in at least three nodes inside the observable range $R_{\mathrm{CMB}}$. Anything less will not qualify as an oscillation, and the corrections will at most show up as a spectral tilt or perhaps a running, or in the worst case, as a normalisation. Depending on the phase $\phi$ three nodes is equivalent to $(1 \div 5 / 4) L$, where $L$ is the oscillation length defined immediately after equation (3.1). This then sets an upper limit on the range of $\xi / \epsilon$,

$$
\frac{\xi}{\epsilon} \lesssim \frac{4}{5 \pi} R_{\mathrm{CMB}} \simeq \begin{cases}1.8, & \text { WMAP }, \\ 2.0, & \text { Planck and CVL }\end{cases}
$$

in which some vaguely oscillatory effect may be observable.

A lower bound on $\xi / \epsilon$ arises from the finite widths of the CMB "window functions". The CMB anisotropy spectra can be written schematically as

$$
C_{\ell}^{i j}=\sum_{\alpha=s, t} \int d \ln k T_{\ell}^{i, \alpha}(k) T_{\ell}^{j, \alpha}(k) P_{\alpha}(k),
$$

where $i, j=T, E, B$, and $\alpha$ sums over scalar and tensor modes. If the width of $T_{\ell}^{i, \alpha}(k) T_{\ell}^{j, \alpha}(k)$ is much larger than the oscillation length $L$, then we expect the oscillations to be wiped out, or, at least, suppressed by a factor of a few. For the $\Lambda$ CDM model, the log width of $T_{\ell}^{i, \alpha}(k) T_{\ell}^{j, \alpha}(k)$ at $\ell \sim 1000$ is $\sim 0.07$, and increases with decreasing $\ell$. Therefore, in order to discern an oscillatory signature, we must have

$$
\frac{\xi}{\epsilon} \gtrsim 0.022 \text {. }
$$

We note that the window functions for the LSS power spectrum from present galaxy redshift surveys are even broader. For the power spectrum of the Sloan Digital Sky Survey Luminous Red Galaxy (SDSS LRG) sample published in reference [38], the window functions have a $\log$ width of $\sim 0.33$, leading to $\xi / \epsilon \gtrsim 0.1$. This means that present galaxy redshift surveys are not very useful for resolving small ripples. 


\subsection{Constraints on the oscillation amplitude}

Suppose the true primordial power spectrum does have trans-Planckian ripples. One can then ask, how large does $\xi$ need to be so that a smooth spectrum is a "bad" fit to the data. This can be estimated as follows.

The effective $\chi^{2}$ for a cosmic variance-limited CMB experiment in one mode (e.g., $T T$ ) is given by

$$
\chi_{\text {eff }}^{2}=f_{\text {sky }} \sum_{\ell=2}^{\ell_{\max }}(2 \ell+1)\left(\frac{C_{\ell}^{\text {obs }}}{C_{\ell}}+\ln \frac{C_{\ell}}{C_{\ell}^{\text {obs }}}\right),
$$

where $C_{\ell}^{\text {obs }}$ denotes the observed $\mathrm{CMB}$ anisotropy spectrum, $C_{\ell}$ the theoretical predictions, and we have included a factor $f_{\text {sky }} \simeq 0.7$ to account for the inevitable sky cut. The difference in $\chi_{\text {eff }}^{2}$ between the smooth and the oscillatory models is then

$$
\Delta \chi_{\text {eff }}^{2}=f_{\text {sky }} \sum_{\ell=2}^{\ell_{\max }}(2 \ell+1)\left(\frac{C_{\ell}^{\text {obs }}}{C_{\ell}^{\text {smooth }}}-\frac{C_{\ell}^{\text {obs }}}{C_{\ell}^{\text {osc }}}+\ln \frac{C_{\ell}^{\text {smooth }}}{C_{\ell}^{\text {osc }}}\right),
$$

where $C_{\ell}^{\text {smooth }}$ and $C_{\ell}^{\text {osc }}$ denote the theoretical predictions of the two respective models.

We express the oscillatory anisotropy spectrum $C_{\ell}^{\text {osc }}$ in terms of the smooth one as

$$
C_{\ell}^{\text {osc }}=C_{\ell}^{\text {smooth }}\left(1+\Delta_{\ell}\right),
$$

where $\Delta_{\ell} \ll 1$ contains the oscillatory features and in general must be calculated by folding in the appropriate transfer functions with the primordial power spectrum (2.21). Here, we estimate its effects by substituting $\Delta_{\text {rms }}$ for $\Delta_{\ell}$, where

$$
\Delta_{\mathrm{rms}}=\frac{\xi}{\sqrt{2}}
$$

is the rms amplitude. Then, keeping only terms to the lowest order in $\xi$, we get

$$
\begin{aligned}
\Delta \chi_{\mathrm{eff}}^{2} & \simeq \frac{\xi^{2}}{4} f_{\mathrm{sky}} \sum_{\ell=2}^{\ell_{\max }} 2 \ell+1 \\
& =\frac{\xi^{2}}{4} f_{\mathrm{sky}}\left(\ell_{\max }+3\right)\left(\ell_{\max }-1\right) \simeq \frac{\xi^{2}}{4} f_{\mathrm{sky}} \ell_{\max }^{2},
\end{aligned}
$$

where we have also used the expectation value $\left\langle C_{\ell}^{\text {obs }}\right\rangle=C_{\ell}^{\text {osc }}$.

As said, the expression (3.10) assumes we are measuring in one mode only (e.g., $T T$ ). To account, approximately, for additional modes (e.g., $T E, E E$ ), we need only to sum their contributions to $\Delta \chi_{\text {eff }}^{2}$. We note that this procedure is strictly not correct since the $T T, T E$ and $E E$ modes in principle correlated, and a simple summation would tend to overestimate $\Delta \chi_{\text {eff }}^{2}$. However, since we are interested only in order magnitude estimates, a small overestimation is not a major concern.

Suppose our TT measurements are cosmic variance-limited up to $\ell_{\max }=2000$ (e.g, Planck). Then a " $2 \sigma$ " detection of trans-Planckian ripples, i.e., $\Delta \chi_{\text {eff }}^{2} \gtrsim 4$, would require

$$
\xi \gtrsim 0.0024
$$

If in addition $T E$ and $E E$ are cosmic variance-limited up to the same $\ell_{\max }$,

$$
\xi \gtrsim 0.0014 \text {. }
$$




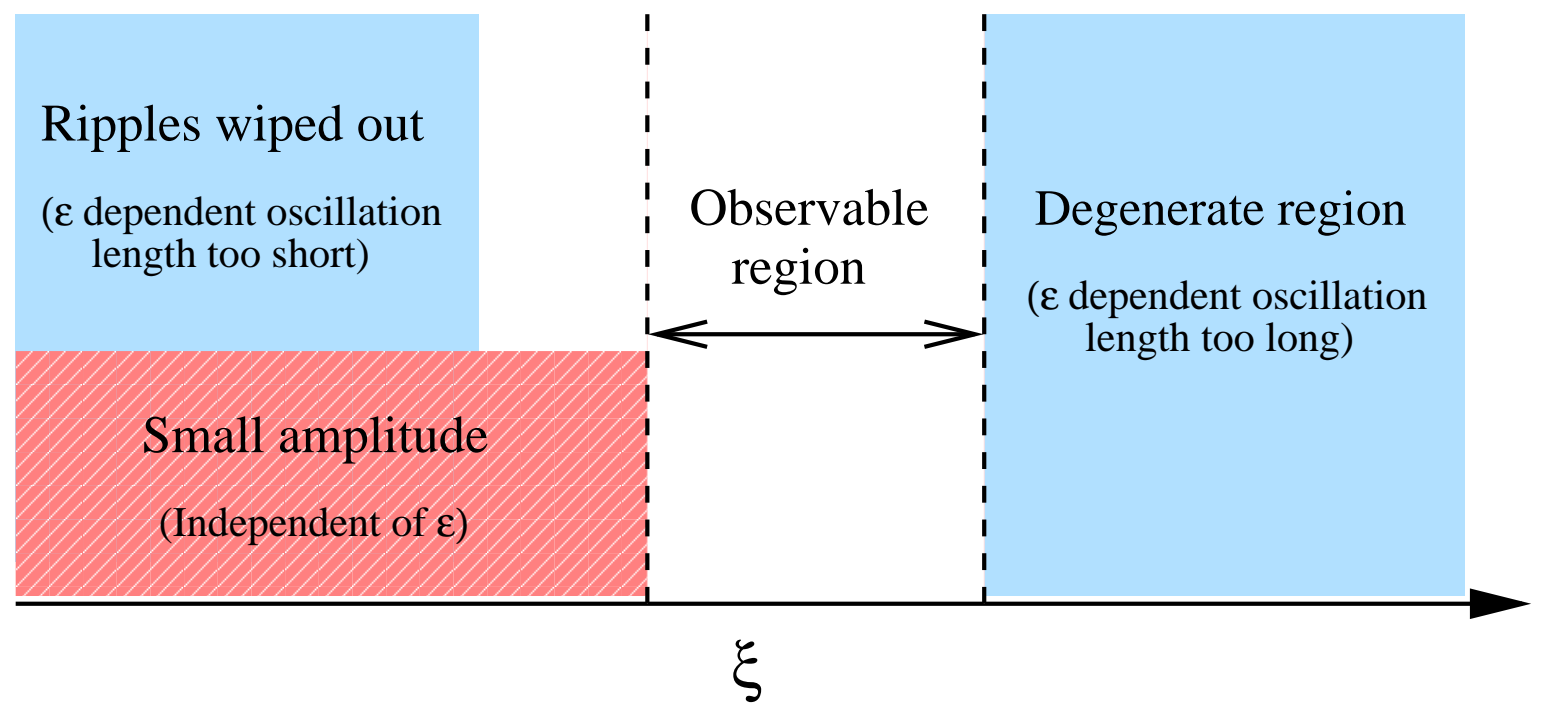

Figure 1. Schematic showing the observability of trans-Planckian ripples. The blue/solid box on the right represents the region in which the oscillation length is so long that $\xi$ becomes degenerate with other parameters describing the primordial power spectrum. The blue/solid box on the left denotes the region in which the ripples are wiped out by the window functions of the experiment because of too short an oscillation length. The red/hatched box on the left encompasses the region in which the oscillation amplitude is too small compared with the sampling error (i.e., cosmic variance). Two vertical dashed lines demarcate the only window in which trans-Plankian ripples may be detectable.

The corresponding limit for a WMAP-like measurement is

$$
\xi \gtrsim 0.0096,
$$

noting that the WMAP TT measurement is effectively noise-free only up to $\ell_{\max } \simeq 500$.

Finally, in deriving these amplitude limits we have assumed that $\xi$ is not degenerate with other cosmological parameters. This is a good approximation if $\xi$ satisfies the bounds (3.3) and (3.5) on the oscillation length. In other words, if trans-Planckian effects manifest themselves as oscillations in the observable range, then no other parameter describing the primordial power spectrum can mimic their effects on the cosmological observables. Trans-Planckian ripples might conceivably be confused with baryon acoustic oscillations (see reference [39] for a related scenario where this is the case). We do not, however, find this to be a problem since the ripples are oscillations in $\ln k$, while the baryon acoustic oscillations are oscillations in $k$. Results from our numerical parameter error forecasts corroborate this claim.

\section{Can we detect ripples?}

A simple picture emerges from the above considerations (figure 1). For a given $\epsilon$, oscillation length requirements define a window in $\xi$ in which trans-Planckian ripples are potentially observable. Amplitude requirements define a second, $\epsilon$-independent window. 
Table 1. Ranges of $\xi$ in which trans-Planckian effects can be observed as oscillations for WMAP, Planck, and CVL for fixed values of $\epsilon$. The parentheses denote those cases that are in reality unobservable, either because of an undetectably small $\epsilon$, or because the expected measurement error on $\xi$ exceeds the width of the detection window.

\begin{tabular}{lccc}
\hline$\epsilon$ & WMAP & Planck & CVL \\
\hline 0.02 & $(0.0096 \lesssim \xi \lesssim 0.036)$ & $0.0024 \lesssim \xi \lesssim 0.041$ & $0.0014 \lesssim \xi \lesssim 0.041$ \\
0.01 & $(0.0096 \lesssim \xi \lesssim 0.018)$ & $0.0024 \lesssim \xi \lesssim 0.020$ & $0.0014 \lesssim \xi \lesssim 0.020$ \\
0.001 & - & - & $(0.0014 \lesssim \xi \lesssim 0.0020)$ \\
0.0001 & - & - & - \\
\hline
\end{tabular}

Only when the two windows overlap can one expect to see ripples. Table 1 summarises the $\xi$ ranges in which ripples can be observed for WMAP, Planck, and a CMB survey that is cosmic variance-limited in $T T, T E$ and $E E$ up to $\ell_{\max }=2000(\mathrm{CVL})$ for various fixed values of $\epsilon$.

Clearly, a crucial prerequisite for ripples detection is that $\epsilon$ must be sizeable. With $\epsilon$ fixed at a value as large as $\gtrsim 0.01$, WMAP, Planck, and CVL all have the potential to see trans-Planckian ripples, although the observable window for WMAP is so small that taking into account the expected measurement error on $\xi$,

$$
\Delta \xi \sim \xi_{\min },
$$

where $\xi_{\min }$ denotes the minimum detectable $\xi$, would most likely close it. If we fix $\epsilon=0.001$, ripples are unobservable for WMAP and Planck, but are in principle still in range for CVL (barring the finite $\Delta \xi$ ). No detection window exists for $\epsilon=0.0001$ or smaller, since the associated oscillation lengths are so large that $\xi$ always falls in the region degenerate with the spectral tilt and/or the normalisation.

The question then is, how well do we expect to measure $\epsilon$ from CMB data, since by the same arguments any measurement of $\epsilon$ that is consistent with $\epsilon=0$ means that no window exists for the detection of trans-Planckian ripples. A null detection of $\epsilon$ by WMAP, $\epsilon \lesssim 0.02$ (95\% C.L.), essentially rules out the observation of ripples by the current generation of CMB probes. The projected $1 \sigma$ sensitivities to $\epsilon$ for Planck and CVL from our parameter error forecasts (see Appendix A for details) are $\Delta \epsilon \simeq 0.005$ and $\Delta \epsilon \simeq 0.0015$ respectively for an undetectable $\xi$. Some variations exist if there is a detection of $\xi$ (empirically, $\Delta \epsilon / \epsilon \sim \Delta \xi / \xi$ ). In table 1, we enclose in parentheses those scenarios that are most likely unobservable either because of an undetectably small $\epsilon$, or because the expected error on $\xi$ exceeds the width of the detection window.

To confirm our analytic estimates we perform several rigorous parameter error forecasts. Figure 2 shows the $\Delta \chi_{\text {eff }}^{2}$ curves for three different fiducial models, assuming the CVL experiment. Technical details of the forecast and definitions of the statistical quantities can be found in Appendix A.

For fiducial model (i) with $\xi_{\text {fid }}=0.01$ and $\epsilon_{\text {fid }}=0.01$, the prospects for ripples detection are excellent. An $\epsilon_{\text {fid }}$ so large can be pinned down by CVL at better than $5 \sigma$. This enables a detection of $\xi$ with a correspondingly high statistical significance: between 


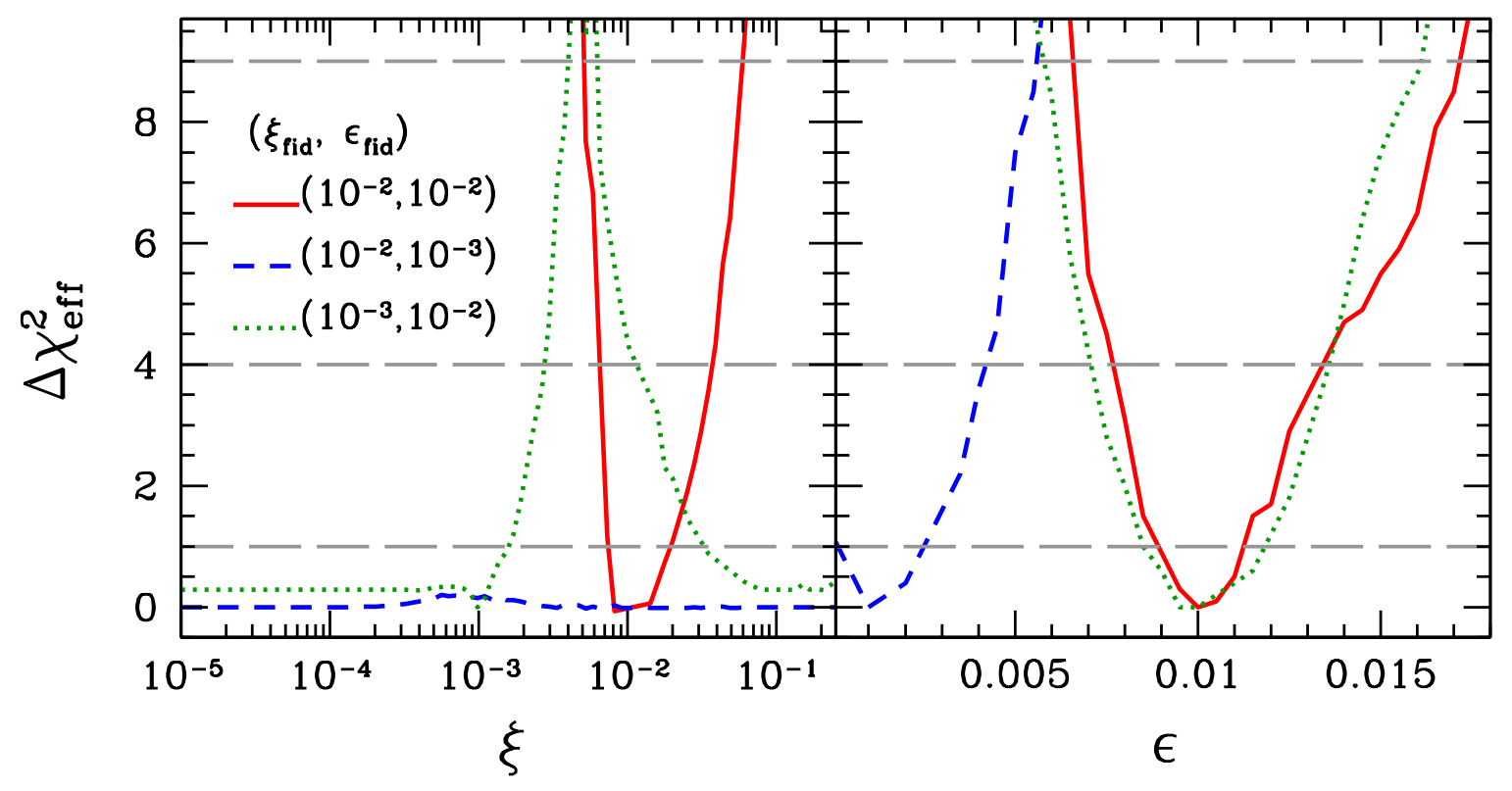

Figure 2. $\Delta \chi_{\text {eff }}^{2}$ curves as functions of $\xi$ and $\epsilon$ for three different fiducial models for the CVL experiment: (i) $\xi_{\text {fid }}=0.01, \epsilon_{\text {fid }}=0.01$ (red/solid), (ii) $\xi_{\text {fid }}=0.01, \epsilon_{\text {fid }}=0.001$ (blue/dashed), and (iii) $\xi_{\text {fid }}=0.001, \epsilon_{\text {fid }}=0.01$ (green/dotted). The three horizontal lines indicate, from top to bottom, $\Delta \chi_{\text {eff }}^{2}=9,4,1$ (i.e., $3 \sigma, 2 \sigma, 1 \sigma$ ).

the best-fit $\xi$ and at $\xi \rightarrow 0$ (i.e., a smooth spectrum), we find $\Delta \chi_{\text {eff }}^{2} \sim 40$. However, if $\epsilon_{\text {fid }}$ is lowered to 0.001 (model (ii)), no detection of $\xi$ is possible, since $\epsilon=0.001 \pm 0.0015$ ( $1 \sigma$ ) is consistent with $\epsilon=0$ so that $\xi_{\text {fid }}=0.01$ lies in the degenerate region.

Model (iii) with $\xi_{\text {fid }}=0.001$ and $\epsilon_{\text {fid }}=0.01$ is also futile, even though the projected $1 \sigma$ error on $\epsilon, \Delta \epsilon \simeq 0.0015$, makes $\epsilon_{\text {fid }}$ well within experimental reach. The reason here is that $\xi_{\text {fid }}=0.001$ leads to an unobservably small oscillation amplitude. Interestingly, although the $\xi_{\text {fid }}$ of model (iii) is beyond CVL's reach, the very large and detectable $\epsilon_{\text {fid }}$ still makes it possible to exclude a range of $\xi$ values from observations. This is in contrast with model (ii), for which an undetectably small $\epsilon_{\text {fid }}$ means that no conclusions can be drawn about $\xi$ (to be discussed in more detail in section 5).

The results of figure 2 are in excellent agreement with our analytic estimates presented in table 1 . Using the same arguments we can also conclude that if $\xi_{\text {fid }}=0.01$ is to be detected by CVL, the underlying model must have $\epsilon_{\text {fid }} \gtrsim 0.005$. This result is fully consistent with a similar claim by Easther, Kinney and Peiris (EKP) [27], who found that $\xi^{\mathrm{EKP}}=0.01$ is detectable at $2 \sigma$ by a CMB experiment that is cosmic variancelimited in TT, TE, EE and $B B$ up to $\ell_{\max }=1500$ only if $r \gtrsim 0.1$ (or $\epsilon \gtrsim 0.006$ ). $\S$

$\S$ EKP used a power spectrum slightly different from our equation $(2.21): P(k)=P^{B D}(k)(\cdots)^{1 / 4}$. This means our $\xi / \epsilon$ constraints from oscillation length considerations apply also to $\xi^{\mathrm{EKP}} / \epsilon$, while our lower limits on $\xi$ from amplitude arguments need to be multiplied by a factor of four for use with $\xi^{\mathrm{EKP}}$. 


\section{Can we constrain ripples?}

Suppose we detect $\epsilon=0.01$, and the true primordial power spectrum has no transPlanckian ripples. Then using the same amplitude arguments as those in section 3.2, we can put a $2 \sigma$ constraint on $\xi$ of

$$
\xi \lesssim 0.0014
$$

for the CVL experiment. If we take instead the EKP ripples and experimental set-up, the same procedure gives a $2 \sigma$ limit of $\xi^{\mathrm{EKP}} \lesssim 0.0064$, which is in good agreement with EKP's $\xi^{\text {EKP }} \lesssim 0.004$ for a scenario with $r=0.15$.

However, this upper limit is not the whole story. This is because if $\xi$ is very large $(\xi \gg \epsilon)$, then for any given $\epsilon \ll 1$, there exists an exact degeneracy between $\xi$ and $\phi$. To see this we perform a small $x \equiv(2 \epsilon / \xi) \ln \left(k / k_{0}\right)$ expansion of the sine function in equation (3.1). This gives us a set of effective parameters

$$
\begin{aligned}
& A_{s, t}^{\mathrm{eff}}=A_{s}\left(1+\xi \sin \phi^{\prime}\right), \\
& n_{s, t}^{\mathrm{eff}}=n_{s, t}+\frac{\epsilon\left(2 \cos \phi^{\prime}-\xi \sin \phi^{\prime}\right)}{1+\xi \sin \phi^{\prime}}, \\
& \alpha_{s}^{\mathrm{eff}}=\alpha_{s}+\frac{2 \epsilon\left(n_{s}-1\right)\left(2 \cos \phi^{\prime}-\xi \sin \phi^{\prime}\right)}{1+\xi \sin \phi^{\prime}}, \\
& \alpha_{t}^{\mathrm{eff}}=\alpha_{t}+\frac{2 \epsilon n_{t}\left(2 \cos \phi^{\prime}-\xi \sin \phi^{\prime}\right)}{1+\xi \sin \phi^{\prime}} .
\end{aligned}
$$

Applying the consistency relations $A_{t}^{\mathrm{eff}} / A_{s}^{\mathrm{eff}}=A_{t} / A_{s}=16 \epsilon$ and $n_{t}^{\mathrm{eff}}=n_{t}=-2 \epsilon$, we see that for whatever $\xi$ and $\epsilon$ we choose, setting $\phi^{\prime}=\arctan (2 / \xi)$ always returns the exact solutions $A_{s, t}=A_{s, t}^{\mathrm{eff}}, n_{s, t}=n_{s, t}^{\mathrm{eff}}$, and $\alpha_{s, t}=\alpha_{s, t}^{\mathrm{eff}}$.

More (approximate) solutions exist if we ignore the consistency constraints on $n_{t}^{\text {eff }}$ and $\alpha_{t}^{\text {eff }}$ (a good approximation since future data are unlikely to resolve the tensor power spectrum), or if the running of the scalar spectral tilt $\alpha_{s}$ is treated also as a free parameter. Thus, unless we have a reason to fix $\left(\phi, n_{s}, A_{s}\right)$ to some particular values, it is always possible to find a fit in the large $\xi \gg \epsilon$ limit that is almost or even exactly as good as that of a smooth spectrum. For this reason a lower limit on large $\xi$ should exist in addition to the upper limit (5.1) on small $\xi$.

Unfortunately there is no simple way to estimate this lower limit accurately, since before we enter the exactly degenerate region at $\xi \gg \epsilon$, there exists a region at $\xi \sim \epsilon$ in which $\xi$ already exhibits some (partial) degeneracy with other parameters describing the primordial power spectrum. The small $x$ expansion limits the exactly degenerate region to $x \lesssim 1$, or

$$
\frac{2 \epsilon}{\xi} \ln \left(\frac{k}{k_{0}}\right) \sim \frac{2 \epsilon}{\xi} R_{\mathrm{CMB}} \lesssim 1, \quad \Rightarrow \quad \xi \gtrsim 18 \epsilon
$$

for the CVL experiment. A less stringent limit can be set by the same oscillation length arguments that led to equation (3.3), i.e.,

$$
\xi \gtrsim 2.0 \epsilon,
$$




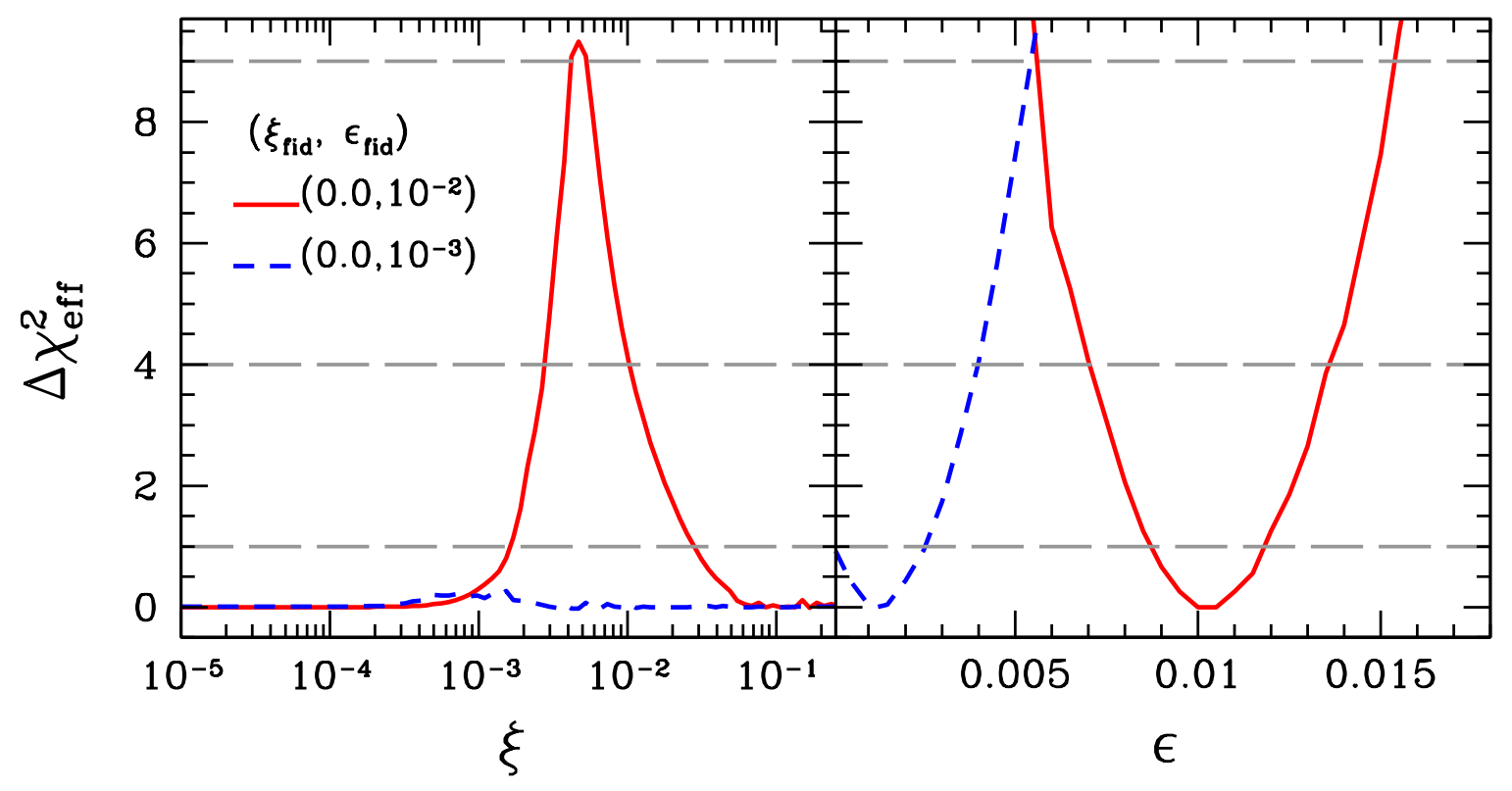

Figure 3. Same as figure 2, but for the models (i) $\xi_{\text {fid }}=0.0, \epsilon_{\text {fid }}=0.01$ (red/solid), and (ii) $\xi_{\text {fid }}=0.0, \epsilon_{\text {fid }}=0.001$ (blue/dashed).

again for CVL. The true limit likely lies somewhere in between (5.3) and (5.4).

For $\epsilon \gtrsim 0.01$, these lower limits on large $\xi$ are purely academic since the transPlanckian spectrum used here assumes $\xi \ll 1$. They are important, however, in the case of small $\epsilon$, since the lower limit (5.3) or (5.4) may run into the upper limit (5.1), thereby closing the window in which some finite $\xi$ may be a "bad fit" to the data. For example, no window exists for the case of a fixed $\epsilon=0.0001$. In the same vein, if $\epsilon=0$ is consistent with data, then we can draw no conclusions about $\xi$.

Figure 3 shows the $\Delta \chi_{\text {eff }}^{2}$ curves for two fiducial models, (i) $\xi_{\text {fid }}=0.0, \epsilon_{\text {fid }}=0.01$, and (ii) $\xi_{\text {fid }}=0.0, \epsilon_{\text {fid }}=0.001$, assuming the CVL experiment. The bounds on $\xi$ for model (i) are in excellent agreement with the predictions (5.1) and (5.4). Model (ii) has an undetectably small $\epsilon_{\text {fid }}$. Correspondingly, no bounds can be set on $\xi$ in this case.

Lastly, we note that EKP found an upper limit on $\xi$ even in the case of an undetectably small $\epsilon$, in apparent contradiction with our results. We believe this to be a consequence of the Bayesian statistics used in their analysis. In the small amplitude region at small $\xi$, all values of the phase $\phi$ provide a good fit to the data, since the oscillations are in any case unobservable. In the degenerate region at larger $\xi$ values, however, only certain choices of $\phi$ give good fits. This reduces the volume of the posterior probability distribution in the $\phi$ direction at large $\xi$ values, which are subsequently disfavoured in a Bayesian analysis.

\section{Galaxy redshift surveys}

Galaxy redshift surveys offer at present the most reliable probe of the large scale structure distribution in the low-redshift universe $(z \lesssim 1)$. Clustering statistics of 
galaxies from the catalogues of the Two-Degree Field Galaxy Redshift Survey (2dFGRS) and the Sloan Digital Sky Survey (SDSS) are now routinely used to constrain cosmological parameters $[38,40]$. Many planned/proposed surveys will probe an even larger volume of the universe and at higher redshifts in the future. Here we consider the potential of the Large Synoptic Survey Telescope (LSST; first light 2015) [31].

The LSST is a full-sky survey looking out to a maximum redshift of $z_{\max } \sim 3$. Within the $\Lambda$ CDM framework this corresponds to a survey volume of $V \sim 100 h^{-3} \mathrm{Gpc}^{3}$. Formally the noise-free region is defined as the range of $k$ values for which the power spectrum signal $P_{k}$ dominates over the Poisson shot-noise. We take this region to be $k \lesssim k_{\max } \sim 0.2 h \mathrm{Mpc}^{-1}$. We caution however that nonlinear evolution such as modecoupling may yet suppress the trans-Planckian ripples or cause them to completely disappear beyond some $k$ value smaller than $k_{\text {max }}$.

The largest scale accessible to the LSST corresponds approximately to $k_{\min } \simeq$ $2 \pi / V^{1 / 3} \simeq 1.4 \times 10^{-3} h \mathrm{Mpc}^{-1}$. The total $k$ range probed by the survey therefore spans barely two orders of magnitude. To maximise the spectral coverage we combine the measurements from the LSST with those from a CMB experiment. Oscillation length arguments then impose an upper limit on $\xi / \epsilon$ for which trans-Planckian ripples can be observed by CMB+LSST,

$$
\frac{\xi}{\epsilon} \lesssim \frac{4}{5 \pi} \ln \left(k_{\max }^{\mathrm{LSST}} / k_{\min }^{\mathrm{CMB}}\right) \simeq 2.1
$$

a number similar to the constraints (3.3) from the CVL experiment alone.

The effective volume of the LSST is some 200 times larger than that of the SDSS LRG sample. Thus we can reasonably expect a corresponding factor of $200^{1 / 3} \sim 6$ decrease in the widths of the $k$-space window functions. The window functions of the SDSS LRG power spectrum has a log width of $\sim 0.33$, from which we derive a log width of $\sim 0.055$ for the LSST. This sets a lower limit on the oscillation length of the trans-Planckian ripples, or, equivalently,

$$
\frac{\xi}{\epsilon} \gtrsim 0.018
$$

a limit comparable to the natural window functions of the CMB (3.5).

To estimate the lowest observable $\xi$ from amplitude arguments we adopt the following expression for the effective $\chi^{2}$ of a sampling variance-limited LSS survey [41]:

$$
\chi_{\mathrm{eff}}^{2}=\frac{V}{(2 \pi)^{2}} \int_{k_{\min }}^{k_{\max }} k^{2} d k\left(\frac{P_{k}-P_{k}^{\mathrm{obs}}}{P_{k}}\right)^{2} .
$$

If the true primordial power spectrum has trans-Planckian ripples, then the $\Delta \chi_{\text {eff }}^{2}$ between a smooth and an oscillatory spectrum is

$$
\Delta \chi_{\text {eff }}^{2}=\frac{V}{(2 \pi)^{2}} \int_{k_{\min }}^{k_{\max }} k^{2} d k \Delta_{k}^{2}
$$

where $\Delta_{k} \ll 1$ encapsulates the oscillatory part of the power spectrum. As in section 3.2, we substitute $\Delta_{k}$ with the rms amplitude $\Delta_{\text {rms }} \equiv \xi / \sqrt{2}$, thus leading to

$$
\Delta \chi_{\text {eff }}^{2} \simeq \frac{V}{(2 \pi)^{2}} \frac{\xi^{2}}{6}\left(k_{\max }^{3}-k_{\min }^{3}\right) \simeq \frac{V}{(2 \pi)^{2}} \frac{\xi^{2}}{6} k_{\max }^{3} .
$$


Table 2. Same as table 1, but for Planck+LSST, and CVL+LSST.

\begin{tabular}{|c|c|c|}
\hline$\epsilon$ & Planck+LSST & CVL+LSST \\
\hline 0.02 & $9.9 \times 10^{-4} \lesssim \xi \lesssim 0.042$ & $8.5 \times 10^{-4} \lesssim \xi \lesssim 0.042$ \\
\hline 0.01 & $9.9 \times 10^{-4} \lesssim \xi \lesssim 0.021$ & 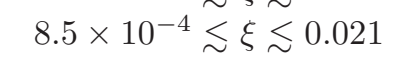 \\
\hline 0.001 & $\left(9.9 \times 10^{-4} \lesssim \xi \lesssim 0.0021\right)$ & $\left(8.5 \times 10^{-4} \lesssim \xi \lesssim 0.0021\right)$ \\
\hline 0.0001 & - & - \\
\hline
\end{tabular}

Adding this $\Delta \chi_{\text {eff }}^{2}$ to its CMB counterpart (3.10) yields the total $\Delta \chi_{\text {total }}^{2}$ for the combination CMB+LSST.

If we demand $\Delta \chi_{\text {total }}^{2} \gtrsim 4$ for a $2 \sigma$ detection, then

$$
\xi \gtrsim \begin{cases}9.9 \times 10^{-4}, & \text { Planck + LSST, } \\ 8.5 \times 10^{-4}, & \text { CVL + LSST } .\end{cases}
$$

Compared with the corresponding limit for Planck alone (3.11), the projected constraint on $\xi$ for Planck+LSST is more than a factor of two better. Adding LSST to CVL also yields some improvement over the limit from CVL alone (3.12). Thus, with the LSST, we will be able to reach down to the $\xi \sim 0.001$ level for the first time. Table 2 summarises the ranges of $\xi$ in which trans-Planckian ripples can be observed for Planck+LSST and CVL+LSST for various fixed values of $\epsilon$.

The remaining question is how sensitive are these combinations of CMB and LSS probes to $\epsilon$. In the case of a non-detection of $\xi$, we find from our parameter error forecasts $1 \sigma$ errors of approximately $\Delta \epsilon \sim 0.0015$ and $\Delta \epsilon \sim 0.005$ for Planck+LSST and CVL+LSST respectively. These are essentially the same sensitivities reachable by Planck and CVL alone. In contrast, a detection of $\xi_{\text {fid }}=0.01$ by Planck + LSST or CVL+LSST does lead to some improvement in the sensitivity to $\epsilon, \Delta \epsilon \sim 0.0007$, for $\epsilon_{\text {fid }}=0.01$. In either case, $\epsilon=0.001$ remains out of reach.

\section{7. $21 \mathrm{~cm}$ surveys}

Recently there is a growing interest to map the three-dimensional distribution of neutral hydrogen via the observation of brightness temperature fluctuations in the $21 \mathrm{~cm}$ spin-flip transition line. A number of radio arrays have been planned/proposed for this observation, targeting specifically the redshift range $6 \lesssim z \lesssim 20$ [32-34]. At these redshifts spatial fluctuations of the $21 \mathrm{~cm}$ signal are in general determined by a combination of the underlying matter distribution and reionisation physics. However if the signal originates at a time when the neutral hydrogen spin temperature is much larger than the CMB temperature (i.e., $z \lesssim 10$ ) and if at the same time the intergalactic medium is completely neutral, the $21 \mathrm{~cm}$ spatial fluctuations are an exact tracer of the perturbations in the underlying matter density field up to a bias factor. It is within this very optimistic scenario that we consider the detectability of trans-Planckian ripples by $21 \mathrm{~cm}$ surveys. See reference [42] for a review on the physics of the $21 \mathrm{~cm}$ transition. 
Observing trans-Planckian ripples

Table 3. Same as table 1, but for Planck+MWA, Planck+SKA, and CVL+FFTT.

\begin{tabular}{lccc}
\hline$\epsilon$ & Planck+MWA & Planck+SKA & CVL+FFTT \\
\hline 0.02 & $0.0024 \lesssim \xi \lesssim 0.040$ & $0.0014 \lesssim \xi \lesssim 0.048$ & $7.9 \times 10^{-5} \lesssim \xi \lesssim 0.050$ \\
0.01 & $0.0024 \lesssim \xi \lesssim 0.020$ & $0.0014 \lesssim \xi \lesssim 0.024$ & $7.9 \times 10^{-5} \lesssim \xi \lesssim 0.025$ \\
0.001 & - & $(0.0014 \lesssim \xi \lesssim 0.0024)$ & $7.9 \times 10^{-5} \lesssim \xi \lesssim 0.0025$ \\
0.0001 & - & - & $\left(7.9 \times 10^{-5} \lesssim \xi \lesssim 2.5 \times 10^{-4}\right)$ \\
\hline
\end{tabular}

Because they observe at high redshifts, $21 \mathrm{~cm}$ surveys tend to have large effective volumes and can in principle probe larger wavenumbers $k$ of the LSS power spectrum without impediments from nonlinear evolution. However, these surveys are limited at the low $k$ end of the spectrum because of residual foregrounds. Typically one expects the foreground to swamp out the $21 \mathrm{~cm}$ signal at $k \lesssim 0.07 h \mathrm{Mpc}^{-1}$. Thus, it is necessary to combine $21 \mathrm{~cm}$ with CMB observations to optimise $k_{\min }$.

To estimate $k_{\max }$ we use figure 3 of reference [43] which shows the effective volumes of the Murchison Widefield Array (MWA) [32], the Square Kilometre Array (SKA) [33], and the Fast Fourier Transform Telescope (FFTT) [34]. We define $k_{\max }$ as the point at which the effective volume drops below one half of the maximum effective volume for each survey (effectively where the thermal noise of the radio telescope becomes comparable to the $21 \mathrm{~cm}$ signal). For MWA, SKA and FFTT, we find $k_{\max } \sim 0.15,0.6,1 h \mathrm{Mpc}^{-1}$ respectively. Oscillation length arguments then lead to the bounds

$$
\frac{\xi}{\epsilon} \lesssim \frac{4}{5 \pi} \ln \left(k_{\max }^{21 \mathrm{~cm}} / k_{\min }^{\mathrm{CMB}}\right) \simeq \begin{cases}2.0, & \mathrm{CMB}+\mathrm{MWA}, \\ 2.4, & \mathrm{CMB}+\mathrm{SKA}, \\ 2.5, & \mathrm{CMB}+\mathrm{FFTT} .\end{cases}
$$

To estimate the lowest observable $\xi$ by our $21 \mathrm{~cm}$ surveys we use again the $\Delta \chi_{\text {eff }}^{2}$ expression (6.5), but replace $V$ with the effective volumes from figure 3 of reference [43] (i.e., $V \sim 1.5 h^{-3} \mathrm{Gpc}^{3}$ for MWA and SKA, and $V \sim 150 h^{-3} \mathrm{Gpc}^{3}$ for FFTT). Again, we compute $\Delta \chi_{\text {total }}^{2}$ by adding $\Delta \chi_{\text {eff }}^{2}$ to its CMB counterpart. Demanding that $\Delta \chi_{\text {total }}^{2} \gtrsim 4$ then gives

$$
\xi \gtrsim \begin{cases}0.0024, & \text { Planck + MWA } \\ 0.0014, & \text { Planck + SKA } \\ 7.9 \times 10^{-5}, & \text { CVL + FFTT. }\end{cases}
$$

Table 3 contains a summary of the $\xi$ ranges detectable by Planck + MWA, Planck + SKA, and CVL+FFTT.

With a potential to reach down to $\xi \lesssim 10^{-4}$, CVL+FFTT improves the sensitivity of CVL alone by a factor of 20. That CVL and FFTT observe at different wavenumbers (but with some overlap) also allows their combination to probe, for some given $\epsilon$, larger values of $\xi$ than either experiment alone. Of particular note is that the improved limits will create, for the first time, a sizeable detection window for $\epsilon$ values as small as 0.001 . 


\section{Conclusions}

We have investigated the sensitivity of various future cosmological probes to "ripples" in the primordial power spectrum due to "trans-Planckian" physics. The magnitude of this effect is determined by $\xi=H_{0} / \Lambda$, i.e., the energy scale of inflation relative to the scale of new physics. As also noted in some previous works on the topic (e.g., [22]), the sensitivity to $\xi$ of a given probe is determined by two experimental parameters. One is the precision with which the power spectrum can be measured over the relevant range, specified by the error bars of the data and/or the width of the window functions. The other important parameter is the "lever arm", i.e., the span in $k$-space over which the power spectrum can be measured, which is crucial for disentangling ripples in the power spectrum caused by trans-Planckian physics from a spectral tilt or an amplitude shift.

Qualitatively, the precision of the data is the limiting factor in the case of very small $\xi$, while the lever arm determines, for a given $\epsilon$, the largest $\xi$ that can be detected uniquely as ripples in the primordial power spectrum. The limit imposed by the lever arm also means that the detectability of trans-Planckian ripples depends strongly on the magnitude of the first slow-roll parameter $\epsilon$. If $\epsilon$ is too small, no detection window exists for $\xi$, as can be seen from equation (3.1), as well as more quantitatively from tables 1 to 3 . In the same vein, if $\epsilon=0$ is consistent with data, then no bounds can be set on $\xi$.

We have estimated the detection threshold of these ripples for data from future CMB experiments (Planck and a hypothetical cosmic variance limited experiment CVL), a galaxy redshift survey exemplified by the LSST, and finally a large scale $21 \mathrm{~cm}$ survey. Due to their very low noise and large volumes, both galaxy and $21 \mathrm{~cm}$ surveys will eventually reach a higher precision than CMB observations alone. This will allow us to reach down to smaller values of $\xi$ than ever before. However, large scale structure surveys will not be able to compete with $\mathrm{CMB}$ observations in terms of wavelength coverage. Therefore, in order to maximise the lever arm for the detection of transPlanckian ripples, these surveys should be combined with CMB measurements.

In an optimistic scenario with $\epsilon=0.01$, we find that on the intermediate timescale, data from Planck+LSST will be open a detection window spanning $10^{-3} \lesssim \xi \lesssim 0.02$. In the more distant future the lower limit of the window can possibly be pushed down to $10^{-4}$ using data from a cosmic variance limited CMB experiment combined with the FFTT $21 \mathrm{~cm}$ survey. This same combination of data will also create a window of detection for $\epsilon=0.001$ and possibly lower for the first time $\left(10^{-4} \lesssim \xi \lesssim 0.0025\right)$.

So what are the implications for the scale of new physics $\Lambda$, which is presumably close to or at the Planck scale? Given that present data already constrains the scale of inflation to $H_{0} \lesssim 2 \times 10^{-5} M_{\mathrm{P}}$ [44], we conclude that in the Danielsson model, there are no realistic prospects of ever detecting the traces of new physics in the primordial spectra if $\Lambda \gtrsim 0.2 M_{\mathrm{P}}$. For models in which the amplitude of the ripples is quadratic instead of linear in $\xi$, as predicted by purely effective field theory arguments in reference [18], the new physics would need to set in at energies smaller than $\sim 2 \times 10^{-3} M_{\mathrm{Pl}}$. 
Nonetheless, we have shown that the fantastic precision of future CMB surveys like Planck, and large scale structure surveys such as LSST and FFTT will extend the detection window of trans-Planckian ripples by more than two orders of magnitude compared with present data. After all, the prospect of probing physics close to the Planck scale is a most tantalising one; a detection of trans-Planckian signatures would truly be one of the most important accomplishments of modern precision cosmology.

\section{Acknowledgements}

We acknowledge use of computing resources from the Danish Center for Scientific Computing (DCSC). The work of JH was supported by the ANR (Agence Nationale de la Recherche).

\section{Appendix A. Details of the parameter error forecast}

The cosmological model used in our forecast consists of nine free parameters, the fiducial values of which are as follows: the baryon density $\Omega_{b} h^{2}=0.02273$, dark matter density $\Omega_{c} h^{2}=0.1099$, Hubble parameter $h=0.72$, redshift to reionisation $z_{\text {re }}=12$, scalar spectral index $n_{s}=0.96$ and normalisation $\ln \left(10^{10} A_{s}\right)=3.18$. The fiducial values of the slow-roll parameter $\epsilon$ and the ripple parameter $\xi$ are given in the main text of the paper, while the fiducial phase $\phi$ is 0 . In addition, we use the following consistency relations on the tensor spectral index $n_{t}$ and normalisation $A_{t}: n_{t}=-2 \epsilon$, and $A_{t} / A_{s}=16 \epsilon$.

We generate mock $\mathrm{CMB}$ data using the method discussed in reference [45]. The likelihood function $\mathcal{L}$ is defined as

$$
\chi_{\text {eff }}^{2} \equiv-2 \ln \mathcal{L}=\sum_{\ell=2}^{\ell_{\max }}(2 \ell+1) f_{\text {sky }}\left[\operatorname{Tr}\left(\widetilde{\boldsymbol{C}}_{\ell}^{-1} \hat{\boldsymbol{C}}_{\ell}\right)+\ln \frac{\left|\widetilde{\boldsymbol{C}}_{\ell}\right|}{\left|\hat{\boldsymbol{C}}_{\ell}\right|}-n\right],
$$

where $\hat{\boldsymbol{C}}_{\ell} \doteq \hat{C}_{\ell}^{\mu \nu}, \mu, \nu=T, E$, denotes the mock data covariance matrix, $\widetilde{\boldsymbol{C}}_{\ell} \doteq C_{\ell}^{\mu \nu}+N_{\ell}^{\mu \nu}$ is the total covariance matrix comprising theoretical predictions of the CMB anisotropy spectrum $C_{\ell}^{\mu \nu}$ and the noise power spectrum $N_{\ell}^{\mu \nu}$. The quantity $n$ counts the number of observable modes. For observations in temperature and $E$-type polarisation, $n=2$.

Parameter estimation is performed by $\chi^{2}$-minimisation, using a simulated annealing routine coupled to the Boltzmann code CAMB [46]. We often plot $\Delta \chi_{\text {eff }}^{2}(x)$ as functions of the parameters we wish to constrain (e.g., $x=\xi, \epsilon)$. This is defined as

$$
\Delta \chi_{\mathrm{eff}}^{2}(x) \equiv-2 \ln \left[\frac{\mathcal{L}^{(1)}(x)}{\mathcal{L}_{\max }}\right],
$$

where $\mathcal{L}_{\text {max }}$ is the global maximum of the likelihood function $\mathcal{L}$ defined in (A.1), and

$$
\mathcal{L}^{(1)}(x) \propto \max _{y_{1}, \ldots, y_{N}} \mathcal{L}\left(x, y_{1}, \ldots, y_{N}\right)
$$

is a projection of $\mathcal{L}\left(x, y_{1}, \ldots, y_{N}\right)$ onto the one-dimensional subspace $x$ by maximising along the $y_{1}, \ldots, y_{N}$ directions.

We define our " $1 \sigma$ ", " $2 \sigma$ " and " $3 \sigma$ " intervals as the regions of $x$ satisfying respectively $\Delta \chi_{\text {eff }}^{2} \leq 1,4$, and 9 . We emphasise that these intervals have no formal 
probabilistic interpretation. However, if $\mathcal{L}^{(1)}(x)$ is a Gaussian distribution and we assume flat priors on the model parameters, then these intervals are exactly identical to the $68 \%, 95 \%$, and $99 \%$ credible intervals derived from a Bayesian analysis. See reference [47] for more details on Bayesian versus non-Bayesian interval construction.

\section{References}

[1] A. A. Starobinsky, "A new type of isotropic cosmological models without singularity," Phys. Lett. B 91 (1980) 99.

[2] A. H. Guth, "The Inflationary Universe: A Possible Solution To The Horizon And Flatness Problems," Phys. Rev. D 23 (1981) 347.

[3] A. D. Linde, "A New Inflationary Universe Scenario: A Possible Solution Of The Horizon, Flatness, Homogeneity, Isotropy And Primordial Monopole Problems," Phys. Lett. B 108 (1982) 389.

[4] A. Albrecht and P. J. Steinhardt, "Cosmology For Grand Unified Theories With Radiatively Induced Symmetry Breaking," Phys. Rev. Lett. 48 (1982) 1220.

[5] V. F. Mukhanov, H. A. Feldman and R. H. Brandenberger, "Theory of cosmological perturbations. Part 1. Classical perturbations. Part 2. Quantum theory of perturbations. Part 3. Extensions," Phys. Rept. 215 (1992) 203.

[6] R. Brunetti, K. Fredenhagen and S. Hollands, "A remark on alpha vacua for quantum field theories on de Sitter space," JHEP 0505 (2005) 063 [arXiv:hep-th/0503022].

[7] T. Tanaka, "A comment on trans-Planckian physics in inflationary universe," arXiv:astro$\mathrm{ph} / 0012431$.

[8] A. A. Starobinsky, "Robustness of the inflationary perturbation spectrum to trans-Planckian physics," Pisma Zh. Eksp. Teor. Fiz. 73 (2001) 415 [JETP Lett. 73 (2001) 371] [arXiv:astroph/0104043].

[9] A. A. Starobinsky and I. I. Tkachev, "Trans-Planckian particle creation in cosmology and ultrahigh energy cosmic rays," JETP Lett. 76 (2002) 235 [Pisma Zh. Eksp. Teor. Fiz. 76 (2002) 291] [arXiv:astro-ph/0207572].

[10] E. Keski-Vakkuri and M. S. Sloth, "Holographic bounds on the UV cutoff scale in inflationary cosmology," JCAP 0308 (2003) 001 [arXiv:hep-th/0306070].

[11] M. Porrati, "Bounds on generic high-energy physics modifications to the primordial power spectrum from back-reaction on the metric," Phys. Lett. B 596 (2004) 306 [arXiv:hepth/0402038].

[12] B. R. Greene, K. Schalm, G. Shiu and J. P. van der Schaar, "Decoupling in an expanding universe: Backreaction barely constrains short distance effects in the CMB," JCAP 0502 (2005) 001 [arXiv:hep-th/0411217].

[13] J. Martin and R. H. Brandenberger, "The trans-Planckian problem of inflationary cosmology," Phys. Rev. D 63 (2001) 123501 [arXiv:hep-th/0005209].

[14] R. H. Brandenberger and J. Martin, "The robustness of inflation to changes in super-Planck-scale physics," Mod. Phys. Lett. A 16 (2001) 999 [arXiv:astro-ph/0005432].

[15] U. H. Danielsson, "A note on inflation and transplanckian physics," Phys. Rev. D 66 (2002) 023511 [arXiv:hep-th/0203198].

[16] V. Bozza, M. Giovannini and G. Veneziano, "Cosmological perturbations from a new-physics hypersurface," JCAP 0305 (2003) 001 [arXiv:hep-th/0302184].

[17] M. Giovannini, "Assigning quantum mechanical initial conditions to cosmological perturbations," Class. Quant. Grav. 20 (2003) 5455 [arXiv:hep-th/0308066].

[18] N. Kaloper, M. Kleban, A. Lawrence, S. Shenker and L. Susskind, "Initial conditions for inflation," JHEP 0211 (2002) 037 [arXiv:hep-th/0209231].

[19] J. J. Atick and E. Witten, "The Hagedorn Transition and the Number of Degrees of Freedom of String Theory," Nucl. Phys. B 310 (1988) 291. 
[20] S. F. Hassan and M. S. Sloth, "Trans-Planckian effects in inflationary cosmology and the modified uncertainty principle," Nucl. Phys. B 674 (2003) 434 [arXiv:hep-th/0204110].

[21] L. Bergström and U. H. Danielsson, "Can MAP and Planck map Planck physics?," JHEP 0212 (2002) 038 [arXiv:hep-th/0211006].

[22] Ø. Elgarøy and S. Hannestad, "Can Planck-scale physics be seen in the cosmic microwave background?," Phys. Rev. D 68 (2003) 123513 [arXiv:astro-ph/0307011].

[23] J. Martin and C. Ringeval, "Superimposed Oscillations in the WMAP Data?," Phys. Rev. D 69 (2004) 083515 [arXiv:astro-ph/0310382].

[24] T. Okamoto and E. A. Lim, "Constraining Cut-off Physics in the Cosmic Microwave Background," Phys. Rev. D 69 (2004) 083519 [arXiv:astro-ph/0312284].

[25] J. Martin and C. Ringeval, "Addendum to "Superimposed Oscillations in the WMAP Data?"," Phys. Rev. D 69 (2004) 127303 [arXiv:astro-ph/0402609].

[26] J. Martin and C. Ringeval, "Exploring the superimposed oscillations parameter space," JCAP 0501 (2005) 007 [arXiv:hep-ph/0405249].

[27] R. Easther, W. H. Kinney and H. Peiris, "Observing trans-Planckian signatures in the cosmic microwave background," JCAP 0505 (2005) 009 [arXiv:astro-ph/0412613].

[28] D. N. Spergel et al. [WMAP Collaboration], "Wilkinson Microwave Anisotropy Probe (WMAP) three year results: Implications for cosmology," Astrophys. J. Suppl. 170 (2007) 377 [arXiv:astro$\mathrm{ph} / 0603449]$.

[29] J. Martin and C. Ringeval, "Inflation after WMAP3: Confronting the slow-roll and exact power spectra to CMB data," JCAP 0608 (2006) 009 [arXiv:astro-ph/0605367].

[30] N. E. Groeneboom and Ø. Elgarøy, "Detection of transplanckian effects in the cosmic microwave background," Phys. Rev. D 77 (2008) 043522 [arXiv:0711.1793 [astro-ph]].

[31] Z. Ivezic et al. [LSST Collaboration], "LSST: from Science Drivers to Reference Design and Anticipated Data Products," arXiv:0805.2366 [astro-ph].

[32] http://www.haystack.mit.edu/ast/arrays/mwa/

[33] http://www.skatelescope.org/

[34] M. Tegmark and M. Zaldarriaga, "The Fast Fourier Transform Telescope," arXiv:0805.4414 [astro$\mathrm{ph}]$.

[35] R. Easther, B. R. Greene, W. H. Kinney and G. Shiu, "A generic estimate of trans-Planckian modifications to the primordial power spectrum in inflation," Phys. Rev. D 66 (2002) 023518 [arXiv:hep-th/0204129].

[36] [Planck Collaboration], "Planck: The scientific programme," arXiv:astro-ph/0604069.

[37] M. R. Nolta et al. [WMAP Collaboration], "Five-Year Wilkinson Microwave Anisotropy Probe (WMAP) Observations: Angular Power Spectra," arXiv:0803.0593 [astro-ph].

[38] M. Tegmark et al. [SDSS Collaboration], "Cosmological Constraints from the SDSS Luminous Red Galaxies," Phys. Rev. D 74 (2006) 123507 [arXiv:astro-ph/0608632].

[39] L. Covi, J. Hamann, A. Melchiorri, A. Slosar and I. Sorbera, "Inflation and WMAP three year data: Features have a future!," Phys. Rev. D 74 (2006) 083509 [arXiv:astro-ph/0606452].

[40] S. Cole et al. [The 2dFGRS Collaboration], "The 2dF Galaxy Redshift Survey: Power-spectrum analysis of the final dataset and cosmological implications," Mon. Not. Roy. Astron. Soc. 362 (2005) 505 [arXiv:astro-ph/0501174].

[41] M. Tegmark, "Measuring cosmological parameters with galaxy surveys," Phys. Rev. Lett. 79 (1997) 3806 [arXiv:astro-ph/9706198].

[42] S. Furlanetto, S. P. Oh and F. Briggs, "Cosmology at Low Frequencies: The $21 \mathrm{~cm}$ Transition and the High-Redshift Universe," Phys. Rept. 433 (2006) 181 [arXiv:astro-ph/0608032].

[43] J. R. Pritchard and E. Pierpaoli, "Constraining massive neutrinos using cosmological $21 \mathrm{~cm}$ observations," arXiv:0805.1920 [astro-ph].

[44] W. Valkenburg, L. M. Krauss and J. Hamann, "Effects of Prior Assumptions on Bayesian Estimates of Inflation Parameters, and the expected Gravitational Waves Signal from Inflation," arXiv:0804.3390 [astro-ph]. 
[45] L. Perotto, J. Lesgourgues, S. Hannestad, H. Tu and Y. Y. Y. Wong, "Probing cosmological parameters with the CMB: Forecasts from full Monte Carlo simulations," JCAP 0610 (2006) 013 [arXiv:astro-ph/0606227].

[46] A. Lewis, A. Challinor and A. Lasenby, "Efficient Computation of CMB anisotropies in closed FRW models," Astrophys. J. 538 (2000) 473 [arXiv:astro-ph/9911177].

[47] J. Hamann, S. Hannestad, G. G. Raffelt and Y. Y. Y. Wong, "Observational bounds on the cosmic radiation density," JCAP 0708 (2007) 021 [arXiv:0705.0440 [astro-ph]]. 